\title{
Isotope once discarded as byproduct shows promise as cancer treatment
}

\author{
n Cite as: CMAJ 2018 December 10;190:E1461-2. doi: 10.1503/cmaj.109-5679
}

Posted on cmajnews.com on Nov. 21, 2018.

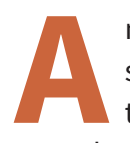
new academic-industry partnership in Canada promises to boost the world's supply of a radioisotope that could improve the treatment of prostate cancer. This agent, actinium 225 (Ac-225), has been a scarce commodity handled by a small circle of facilities scattered around the globe. Although the isotope has never been approved for a medical application, observers have been struck by the reduction of tumours associated with its compassionate use in palliative care.

Researchers are eager to get their hands on enough of the isotope to lay the groundwork for clinical trials, and nuclear laboratories around the world are exploring how to ramp up production. Among those sites is the cyclotron centre TRIUMF in Vancouver, where several years ago administrators realized they were discarding substantial amounts of Ac-225 as a byproduct of other reactions - amounts on the order of the entire global supply.

A collaboration between TRIUMF and Canadian Nuclear Laboratories will attempt to shore up this output, which could become a valuable therapeutic resource. In addition to examining techniques for extracting Ac-225 from the mixture of materials created by other processes, a part of the cyclotron will be dedicated to making the isotope directly.

Canadian Nuclear Laboratories, a subsidiary of Atomic Energy of Canada Ltd. launched in 2014 to support nuclear science and technology initiatives, plans to help TRIUMF with technical challenges. According to Kathryn Hayashi, president and CEO of TRIUMF Innovations, its commercial development arm, these challenges range from basic chemical problems to dealing with government permits that allow radioactive materials to be transported across the country.

"They have complementary expertise and infrastructure to what we have," explains Hayashi, adding that the future of Ac-225 will depend on finding a practical distribution network.

Once that network is in place, one of the first people seeking to use it will be Brigitte Guérin, head of the radiochemistry laboratory at the Sherbrooke Molecular Imaging Centre. She is a principal investigator in a study exploring the use of radioligands in prostate cancer, a project involving 36 scientists and clinicians at five Quebec hospitals.

"We will be working with emerging radionuclides, among them actinium 225," said Guérin, referring to animal research that will set the stage for human testing. "We will be comparing it with

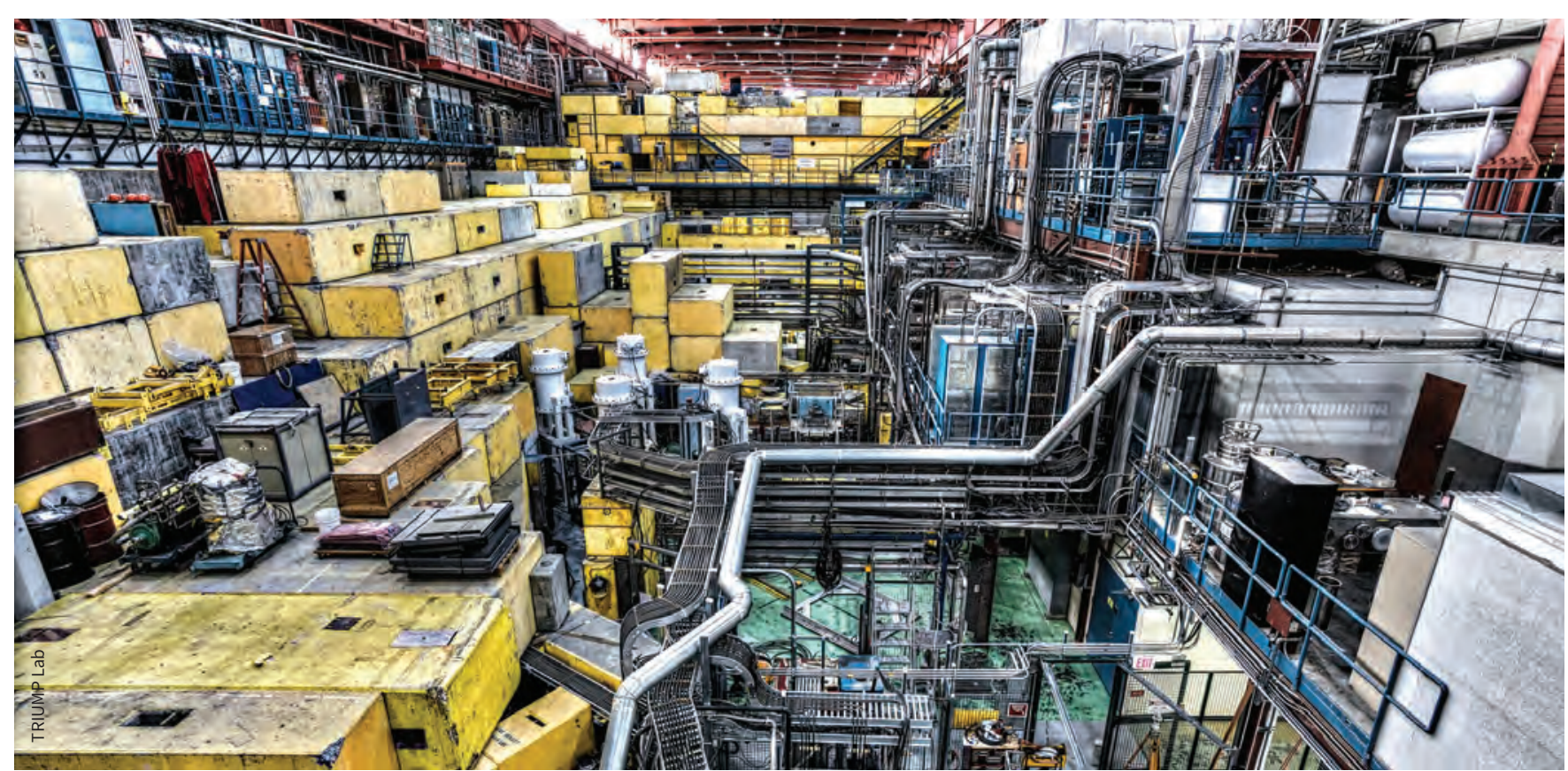

The TRIUMP cyclotron facility in Vancouver is ramping up production of Ac-225. 
lutetium 177 (Lu-177) and other radionuclides that can be used for radiotherapy. To our knowledge, this kind of comparative study has never been done before."

David Laidley of the nuclear medicine department at the London Health Sciences Centre in London, Ontario, welcomes such a comparison. He is working on clinical trials of Lu177 and has been impressed by the ability of that isotope to tackle the most difficult form of prostate cancer, known as "castrate-resistant" because of its persistence even when a patient's testosterone levels have been reduced to low levels.

In contrast to Ac-225, the lutetium isotope is a beta-emitter, sending out high energy particles that can kill a tumour but also travel farther and damage other tissue. Actinium emits alpha particles, which are weaker and travel only a few millimetres before losing potency. In principle, once Ac-225 is conducted to a tumour by attaching it to a prostate-specific membrane antigen, it would affect only the cancerous tissue and cause minimal adverse effects elsewhere.

"There's no reason why there couldn't be a comparison between the two isotopes looking at differences in efficacy," said Laidley, noting that one or both could ultimately become the standard of care in neuroendocrine tumour treatment.

The use of such radioisotopes for both diagnosis and treatment could "revolu- tionize current patient care," according to Frank Wuest of the Cross Cancer Institute in Edmonton, which already treats neuroendocrine tumours with Lu-177. "This will definitely open up a new area where we, together with the physicians, are using regulated drugs in the first stage to visualize the cancer and, more importantly, give an accurate staging of the cancer," he said.

Progress will be driven by basic science around lesser-known isotopes, such as Ac-225, which sets the stage for practical applications, he added. "I'm pretty sure there are more isotopes like actinium that are in the queue waiting to be investigated."

Tim Lougheed, Bath, Ont. 